\title{
Design of Intelligent Charger System
}

\section{Yunting Wu, Weici Liu* and Ruisheng Liang}

\author{
Department of Electronic Information Engineering, Guangzhou College of Technology and \\ Business, Foshan 528138, China
}

${ }^{*}$ Corresponding author

Keywords: Charger, Single-chip microcomputer, Smart.

\begin{abstract}
In this paper, an intelligent charging system which is based on single-chip microcomputer system is introduced. The hardware and software realization of intelligent battery charger based on MCU are presented. The charger studied in this paper has higher practical value and accumulates a lot of practical experience for further research in the future.
\end{abstract}

\section{Introduction}

Nowadays, the rapid charging technology is developing very rapidly and successfully at home and abroad. The batteries can be recharged on regular household current by a high-power charger in a short time. And now with the development of charging technology, the body of charger is getting smaller and smaller, even some high-power chargers can achieve only a bag size.The development of intelligent charger has become the frontier of the research of charger technology. Modern computer technique, power supply technique and fuzzy control theory provide new technical method for research of intelligent charging system. The acceptable charging current at each stage of battery charging keeps a good matching relationship, so that the battery charging process is always in place.In the optimal state, the intelligent charging mode greatly improves the charging quality and efficiency, compared with the conventional charging mode.It can save about $30 \%$ 50\% of the electric energy and open up a new way for the development of new charging technology and charging equipment [1-5].

This paper extensively consults the scientific and technological literature, and through in-depth investigation, designs a battery intelligent charger based on STC89C52 single-chip computer system, which combines the single-chip computer technology and battery charging technology organically. It is a practical charger. The system can achieve the goal of reliability, stability and economy.

It is of great significance to study and design a perfect intelligent charging system. The paper is structured as follows: starting from system model in section II, the system structure and module design are introduced. A brief conclusion is given in section III.

\section{System Model}

The design of battery intelligent charger based on STC89C52 microcontroller system includes microcontroller circuit, charging control circuit, DC power supply design, LM393 (voltage comparator) circuit design, MAX1898 (battery charging management chip) application circuit design, alarm circuit design, etc. The software design includes charging main program and charging termination program design. At the same time, $\mathrm{C}$ language development tools are used for detailed design and coding. The system construction diagram is shown in Figure 1. 


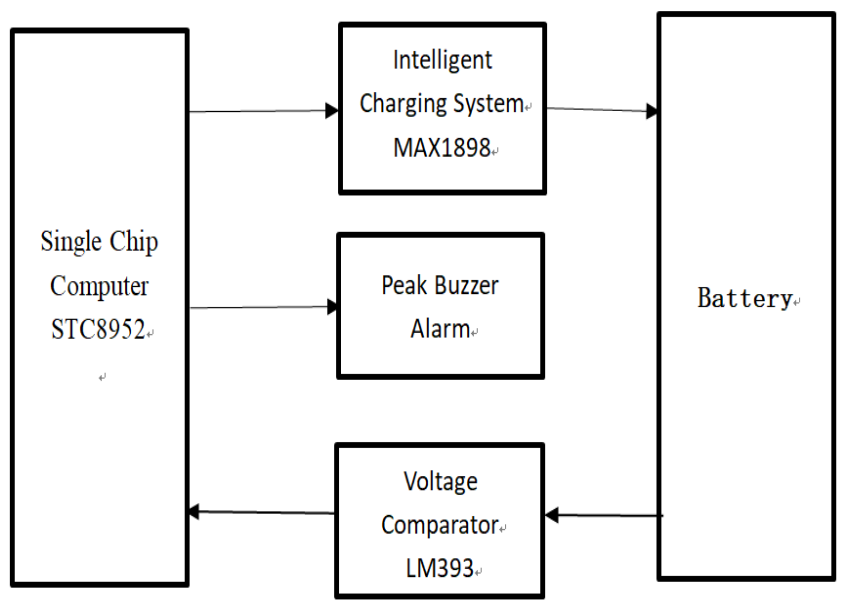

Figure 1. The system construction diagram

\section{Single Chip Computer STC8952}

Single Chip Computer STC8952 has COMS which is 8-bit processor with low power consumption and high cost performance and 8K Flash memory inside the processor. As far as single chip is concerned, STC89C52 provides flexible and effective solutions for many embedded systems.

STC89C52 single-chip computer can complete the inspection and prompt the battery saturation or storage status; at the same time, warn the wrong connection of the battery.

\section{Voltage Comparator LM393}

LM393 voltage comparator can accurately identify and compare two different input signals. Generally, only one voltage is output, up to $2 \mathrm{mv}$. It can also supply power to double power supply. In the process of power supply, electricity consumption will be very little, no matter how much voltage can be achieved. At the same time, in the process of power supply, the input signals of these two independent voltage comparators are almost low voltage signals.

\section{Intelligent Charging System MAX1898}

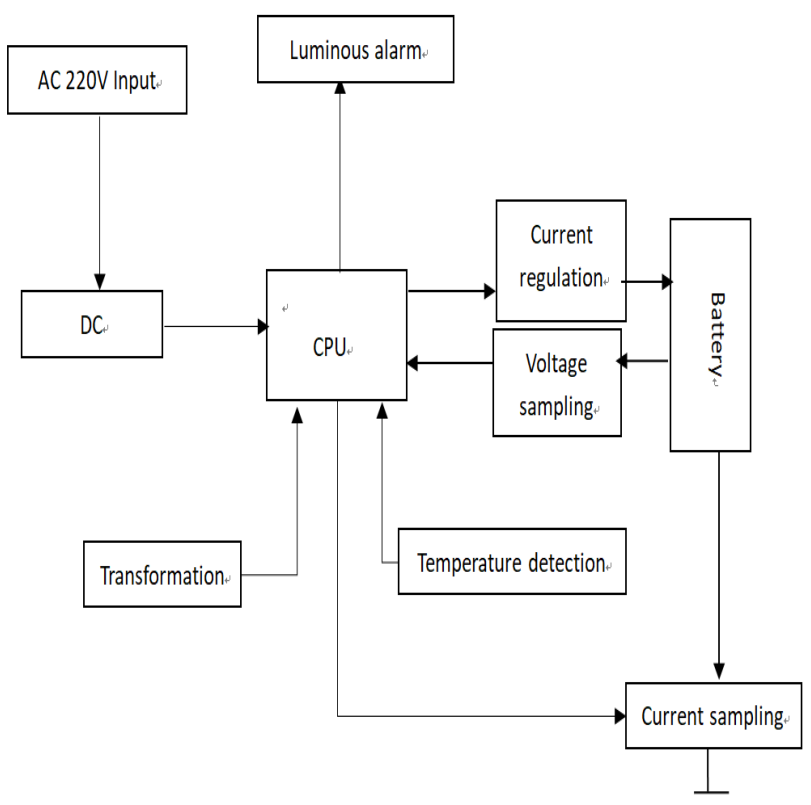

Figure 2. Structural block diagram of intelligent charger

The hardware design of intelligent charger includes not only MCU circuit, but also signal control circuit and charging control circuit. The charging control circuit of MCU circuit achieves the purpose of intelligent control of charger, such as alarm and automatic identification of batteries 
after charging. The design mainly relies on STC89C52 chip and MAX1898 chip which reaches the charge control part. Figure 2 is the structure block diagram of intelligent charger.

\section{Software Design}

The software development of this project is as Figure 3.

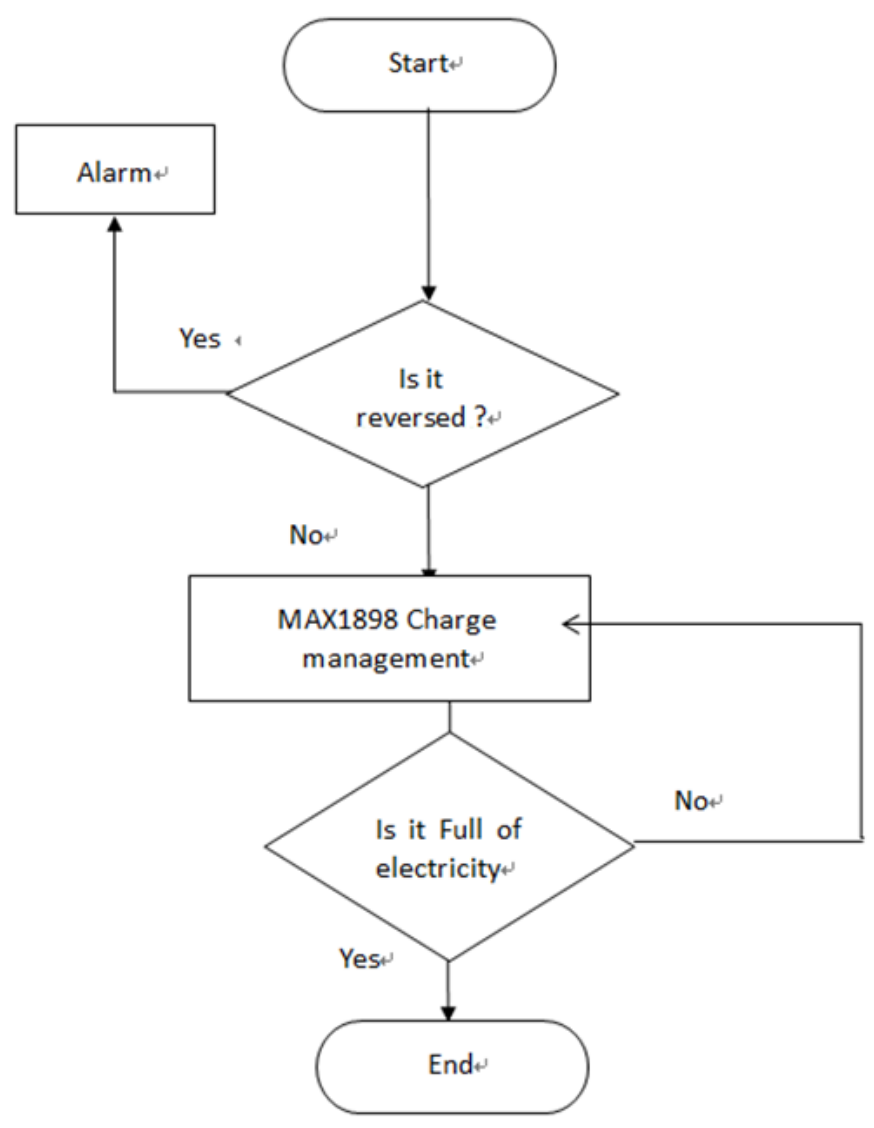

Figure 3. Intelligent charging flow chart

After the smart charger is powered on, the program initializes, and then starts to detect whether the charger terminal is connected to the battery. If it is not connected to the battery, the smart charger will not work. If it detects that it has been connected to the battery, then the smart charger begins to charge the battery. First, the voltage of the battery terminal is judged, and the voltage of the terminal determines the advance charging. If the voltage at the battery end is below the threshold voltage, the smart charger will pre-charge the battery in the form of small current and turn it into the pre-charge state. In this state, if the voltage at the battery end exceeds the threshold voltage, the battery will be charged rapidly in the form of large current. If the battery terminal voltage is above the threshold voltage, the smart charger will jump over the pre-charging state and directly join the situation of rapid charging with high current, and charge the battery within the maximum current allowed. At the same time, the intelligent charger continues to detect the battery terminal voltage. When it detects that the battery terminal voltage exceeds the battery rated voltage, the intelligent charger goes into the overcharging state and charges the battery with a very small current. If the charging time exceeds the set time of the state, the intelligent charger enters the floating charging state, and maintains the battery with a smaller current to make up for the battery. At the same time, the light-emitting diode alarms to inform the battery that charging is over.

\section{Conclusion}

In summary, this design mainly realizes the intelligent charging system based on single chip computer. The intelligent charger introduced in this paper is based on battery charging. The advantage is according to the charging method used by people, it can provide the battery with 
warning services to prevent overvoltage, thermal protection and complete charging. The software used in the design has the characteristics of convenience and high efficiency, and the software is reliable and stable. This intelligent charger can not only solve the compatibility problem between battery and charger efficiently, but also improve the efficiency and safety of battery charging by changing charging parameters to charge different types of batteries. At the same time, the integrated control method is used to judge whether the voltage has negative increment or not, and the temperature and charging time of the battery are used as auxiliary judgment basis. Through these aspects of battery testing, make the charging process more scientific, reasonable and safe.

\section{Acknowledgement}

This research was financially supported by 2019 Natural Science Project of Guangzhou College of Technology and Business (Grants No. KA201931), the Guangdong provincial College Students' innovation and entrepreneurship training program(No. 201813714013), and College Students' innovation and entrepreneurship training program of Guangzhou College of Technology and Business (No. xj201913714079).

\section{References}

[1] Torres-Torriti M, Landau D. Performance Evaluation of UHF RFID Technologies for Real-Time Passenger Recognition in Intelligent Public Transportation Systems[J]. IEEE Transactions on Intelligent Transportation Systems, 2010, 11(3):748-753.

[2] Tanaka T, Sekiya T, Tanaka H, et al. Smart Charger for Electric Vehicles With Power-Quality Compensator on Single-Phase Three-Wire Distribution Feeders[J]. IEEE Transactions on Industry Applications, 2013, 49(6):2628-2635.

[3] Ikeda F , Tanaka T , Yamada H, et al. Smart charger for electric vehicles on single-phase three-wire distribution feeders with constant dc-capacitor voltage control under multiple household customers[C]// International Conference on Electrical Machines \& Systems. IEEE, 2015.

[4] Kei N , Fuka I , Yuki O , et al. Improvement in Harmonic Compensation of a Smart Charger with a Constant DC-Capacitor Voltage-Control-Based Strategy for Electric Vehicles in Single-Phase Three-Wire Distribution Feeders[J]. Energies, 2018, 11(6):1604-.

[5] Mannen T, Fukasawa I, Fujita H. A New Control Method of Suppressing the DC-Capacitor Voltage Ripple Caused by Third-Order Harmonic Compensation in Three-Phase Active Power Filters[J]. Electrical Engineering in Japan, 2018, 202(2):54-62. 